Pacific Journal of Mathematics

GENERIC COVERING PROPERTIES FOR SPACES OF 


\section{GENERIC COVERING PROPERTIES FOR SPACES OF ANALYTIC FUNCTIONS. II}

\section{David A. Stegenga And Kenneth Stephenson}

It is known that for $0<p<\infty$ the Hardy space $H^{p}$ contains a residual set of functions, each of which has range equal to the whole plane at every boundary point of the unit disk. With quite new general techniques, we are able to show that this result holds for numerous other spaces. The space BMOA of analytic functions of bounded mean oscillation, the Bloch spaces, the Nevanlinna space and the Dirichlet spaces $D_{a}$ for $0 \leq a \leq 1 / 2$ are examples. Our methods involve hyperbolic geometry, cluster set analysis and the "depth" function which we have used previously for determining geometric properties of the image surfaces of functions.

Denote by $D(a, r)$ the open disc in $\mathbf{C}$ centered at $a$ and of radius $r$. Denote by $D$ the unit disc $D(0,1)$ and let $\Delta(a, r)=D \cap D(a, r)$ for $a \in \partial D$. Brown and Hansen [4] proved that each Hardy space $H^{p}$, for $0<p<\infty$, contains a residual set of functions $f(z)$ with the property that for each $a \in \partial D$ the range of $f$ at $a$, denoted $R(f, a)$, covers the plane $\mathbf{C}$, with the possible exception of at most a point. Here $R(f, a)=$ $\bigcap\{f(\Delta(a, r)): r>0\}$ is the set of values taken on by $f$ in every neighborhood of $a$. The one point restriction was later removed by others [3].

The techniques used in these papers do not extend to the space of functions of bounded mean oscillation, which we denote as BMOA, nor the space of Bloch functions, which we denote as $B$.

Our paper devoted to generic covering properties of spaces such as $H^{\infty}$ [14] suggested a new approach which would lead to the same conclusion in these and more general situations.

Our setting will be a linear space of analytic functions $M$ defined on the unit disc. $M$ will be an $F$-space, that is a topological vector space with a complete translation invariant metric $d$. We will denote $d(0, f)$ by $\|f\|$ although $\|\cdot\|$ is not in general a norm. We will assume the metric on $M$ induces a topology on $M$ which is finer than that of $H(D)$, i.e., the inclusion map from $M$ to $H(D)$ is continuous. Here $H(D)$ is the usual space of holomorphic functions on the unit disc $D$ with the topology of uniform convergence on compact subsets. 
For $a \in D$, let $b_{a}(z)=z(a-z) /(1-\bar{a} z)$ be a two-fold Blaschke product mapping $D$ onto itself and let $C_{a}: f \rightarrow f \circ b_{a}$ denote the corresponding composition operator on $H(D)$. Let $N$ denote the space of normal functions. As an example of our results we have the following theorem:

THEOREM 1. If $M$ satisfies:

(i) $M \cap N$ is a dense subspace of $M$,

(ii) (a) $\sup \left\{\left\|C_{a} f\right\|, a \in D\right\}<\infty$ for all $f \in M$, and

(b) $M$ contains a function $f$ with $f(D)=\mathbf{C}$, then $\{f \in M: R(f, a)=\mathbf{C}$ for all $a \in \partial D\}$ is a dense $G_{\delta}$-set in $M$.

We observe that the hypothesis (i) is mild since typically $M \cap H^{\infty}$ or $M \cap \mathrm{BMOA}$ is dense in $M$. Certainly this is the case for the Hardy space and BMOA examples. Also, hypothesis (ii)(a) is easily verified for $H^{p}$ or BMOA using subordination. Finally the hypothesis (ii)(b) is a starting point for finding functions which map onto at every boundary point.

Our methods involve hyperbolic geometry, cluster set analysis, and the "depth" function which we have used previously for determining geometric properties of the image surface for a function.

Part of this research took place while the second author held a visiting position at the University of Hawaii and he expresses his appreciation for their fine hospitality. Both authors would like to thank George Csordas for helpful conversations on the material presented here.

1. Preliminaries. The pseudohyperbolic metric $\rho$ is defined on $D$ by $\rho(a, b)=\left|\varphi_{a}(b)\right|$ where $\varphi_{a}(z)=(a-z) /(1-\bar{a} z)$ is a Möbius transformation of $D$ onto $D$. The pseudohyperbolic disc of radius $r>0$, centered at $a \in D$ is denoted by $D_{\rho}(a, r)=\{z \in D: \rho(a, z)<r\}$. By the Schwarz-Pick lemma, if $f$ is an analytic mapping of $D$ into $D$, then $\rho(f(a), f(b)) \leq \rho(a, b)$ for all $a, b \in D$, with equality holding if and only if $f$ is a Möbius transformation. Since $\varphi_{a}^{-1}=\varphi_{a}$ it follows that $\varphi_{a}\left(D_{\rho}(a, r)\right)=D_{\rho}(0, r)=D(0, r)$ for $0<r<1$.

If $G$ is an open connected subset of $\mathbf{C}$, let $H(G)$ denote the space of analytic functions on $G$ with the usual topology of uniform convergence on compact subsets of $G$. For $f \in H(G)$ let $f(G)=\{w=f(z): z \in G\}$. If $G^{\prime} \subset G$ has compact closure in $G$ we write $G^{\prime} \Subset G$. Suppose $f \in H(G)$, $a \in G, b=f(a)$, and $r>0$. Let $\Omega_{a}(r)$ denote the component of $f^{-1}(D(b, r))$ in $G$ which contains $a$. If $\Omega_{a}(r) \Subset G$ then the argument principle implies that there is a positive integer $k$ for which $f$ maps $\Omega_{a}(r)$ $k$-to- 1 onto $D(b, r)$ and $f$ maps $\partial \Omega_{a}(r) k$ times onto $\partial D(b, r)$. 
Definition. Let $G$ be an open connected set in C. If $f \in H(G)$ and $w \in \mathbf{C}$ then the depth function for $f$ on $G$ is defined at $w$ by

$$
\delta(w, f, G)=\sup \left\{r: \Omega_{a}(r) \Subset G \text { for some } a \in f^{-1}(w)\right\} .
$$

We simplify this notation to $\delta_{f}(w)$ or $\delta(w, f)$ when $G$ is implied by the context.

In geometric terms, $\delta_{f}(w)$ is the supremum of the radii of (ramified) discs centered at $w$ which lie in the image surface of $f$. Thus, it tells how deeply $w$ is "buried" in the range of $f$, but in a local sense. The essential properties of $\delta_{f}$ were established in [14]; we repeat the statement here for completeness.

LEMMA 1. Let $G$ be an open connected set in $\mathbf{C}$ and let $f \in H(G)$ be nonconstant. Then

(i) either $\delta_{f}$ is identically equal to $+\infty$ or else is finite at all points,

(ii) $\delta_{f}(w)>0$ if and only if $w \in f(G)$,

(iii) given a compact set $K \subset f(G)$, there is an $\eta>0$ and an open set $G^{\prime} \Subset G$ with $\delta\left(w, f, G^{\prime}\right)>\eta$ for every $w \in K$, and

(iv) given $g \in H(G)$

$$
\sup _{w \in \mathbf{C}}\left|\delta_{f}(w)-\delta_{g}(w)\right| \leq \sup _{z \in G}|f(z)-g(z)|,
$$

with the understanding that the finiteness of the right-hand side implies that $\delta_{f} \equiv+\infty$ only when $\delta_{g} \equiv+\infty$.

The following properties follow immediately from the definition and will be used subsequently.

REMARK 1.

(i) For $w_{0} \in \mathbf{C}, \delta(w, f, G)=\delta\left(w-w_{0}, f-w_{0}, G\right)$.

(ii) If $G_{1} \subset G_{2}$ then $\delta\left(w, f, G_{1}\right) \leq \delta\left(w, f, G_{2}\right)$.

(iii) If $G_{1}, G_{2}$ are plane domains, $\varphi \in H\left(G_{1}\right)$ with $\varphi\left(G_{1}\right)=G_{2}$ and $\varphi$ is a proper mapping on $G_{1}$, then $\delta\left(w, f, G_{2}\right)=\delta\left(w, f \circ \varphi, G_{1}\right)$.

2. Main Theorem. Let $M$ be an $F$-space of analytic functions in $H(D)$ as described in the introduction. Recall that $\|f\|$ denotes the distance in $M$ from $f$ to the zero function.

THEOREM 2. Let $M$ be an F-space in $H(D)$ that satisfies:

(i) for each $a \in \partial D, r>0$, there is a dense subset $M(a, r) \subset M$ with the property that for each $f \in M(a, r)$, either

(a) $R(f, a)=\mathrm{C}$ or 
(b) there is a sequence $\left\{a_{n}\right\}$ in $\Delta(a, r)$ with $a_{n} \rightarrow a^{\prime} \in \partial D,\left|a^{\prime}-a\right|$ $<r$, and there is a $w_{0} \in \mathbf{C}$ with the property that $f \circ \varphi_{a_{n}} \rightarrow w_{0}$ in $H(D)$, and

(ii) for every $R>0, \varepsilon>0$, there exists $r=r(R, \varepsilon)$ in the interval $(0,1)$, and $\eta=\eta(R, \varepsilon)>0$ with the property that: given $a \in D$ there exists $g_{a} \in M$ with $\left\|g_{a}\right\|<\varepsilon$ and such that

$$
\delta\left(w, g_{a} \circ \varphi_{a}, D(0, r)\right) \geq \eta, \quad|w| \leq R .
$$

Then the set, $M_{0}$, of all $f$ in $M$ with $R(f, a)=\mathbf{C}$ for every $a \in \partial D$ is $a$ dense $G_{\delta}$-set in $M$.

Proof. Let $I$ be a countable dense subset of $\partial D$. For $a \in I$ and positive integers $j, k$ set $U(a, j, k)=\left\{f \in M: D(0, k) \Subset f\left(\Delta\left(a, j^{-1}\right)\right)\right\}$. The proof will follow from the Baire category theorem once we establish that $U(a, j, k)$ is a dense open set in $M$; for the countable intersection of the $U(a, j, k)$ 's for $a \in I$, and $j, k=1,2, \ldots$ is precisely $M_{0}$.

In order to prove that $U(a, j, k)$ is an open set in $M$ it suffices to prove that it is relatively open in $H(D)$. Lemma 1 yields a short proof: Let $f \in U(a, j, k), K$ equal to the closure of $D(0, k)$, and $G=\Delta\left(a, j^{-1}\right)$ in Lemma 1 (iii). The lemma provides $\eta>0$ and $G^{\prime} \Subset G$ with $\delta\left(w, f, G^{\prime}\right)$ $>\eta$ for $w \in K$. If $g \in H(D)$ satisfies $|f(z)-g(z)|<\eta$ for $z \in G^{\prime}$ then Lemma 1 (iv) implies that $\delta\left(w, g, G^{\prime}\right)>0$ whenever $w \in K$ and hence Lemma 1 (ii) yields that $D(0, k) \Subset g\left(G^{\prime}\right) \subset g\left(\Delta\left(a, j^{-1}\right)\right)$. Therefore an $\eta-G^{\prime}$ neighborhood of $f$ is contained in $U(a, j, k)$ and it is an open set.

To prove that $U=U(a, j, k)$ is a dense subset let $V$ be an open set in $M$. By (i) there is a function $f$ in $V \cap M\left(a, j^{-1} / 2\right)$. If (i)(a) holds then $f \in U \cap V$ and we are done. We assume therefore that (i)(b) holds and that $a^{\prime}, w_{0}$, and $\left\{a_{n}\right\}$ are obtained from that hypothesis. Let $R=k+\left|w_{0}\right|$ and $\varepsilon>0$. From hypothesis (ii) we get $0<r=r(R, \varepsilon)<1, \eta=\eta(R, \varepsilon)$ $>0$, and for each $b \in D$ we have a function $g_{b} \in M$ with $\left\|g_{b}\right\|<\varepsilon$ and $\delta\left(w, g \circ \varphi_{b}, D(0, r)\right) \geq \eta$ for $|w| \leq R$. By Remark 1 (i), our choice of $R$ implies that

$$
\delta\left(w, g \circ \varphi_{b}-w_{0}, D(0, r)\right) \geq \eta, \quad|w| \leq k .
$$

For $b$ we take $b=a_{n}$ where $n$ is large enough that (by (i)(b))

$$
\sup _{|z| \leq r}\left|f \circ \varphi_{b}(z)-w_{0}\right|<\eta, \quad D_{\rho}(b, r) \subset \Delta\left(a, j^{-1}\right) .
$$

Let $g=g_{b}$ from above and pick $\varepsilon$ small enough so that $f+g$ is still in $V$. The proof will be complete once we show that $f+g \in U$. For this it suffices to show that $D(0, k) \Subset(f+g)\left(D_{\rho}(b, r)\right)$ since $D_{\rho}(b, r) \subset$ $\Delta\left(a, j^{-1}\right)$. But the remark following Lemma 1 along with Lemma 1 (ii) 
makes it plain that we need $\delta\left(w,(f+g){ }^{\circ} \varphi_{b}, D(0, r)\right)>0$ whenever $|w| \leq k$.

Finally, Lemma 1 (iv) and (2) yields:

$$
\begin{aligned}
\left|\delta\left(w,(f+g) \circ \varphi_{b}, D_{\rho}(b, r)\right)-\delta\left(w, g \circ \varphi_{b}-w_{0}, D_{\rho}(b, r)\right)\right| & \\
& \leq \sup _{|z| \leq r}\left|f \circ \varphi_{b}-w_{0}\right|<\eta .
\end{aligned}
$$

Combined with (1) this gives for $|w| \leq k$ that $\delta\left(w,(f+g) \circ \varphi_{b}, D_{\rho}(b, r)\right)$ $>0$ and the proof is complete.

\section{REMARK 2.}

(i) The hypotheses of Theorem 2 need only be satisfied by some dense subspace.

(ii) The continuity of scalar multiplication in an $F$-space was not used in the above proof. Thus, the theorem remains valid for subspaces of $H(D)$ whose topology is determined by a complete translation invariant metric.

3. The proof of Theorem 1. We will need some lemmas in order to establish Theorem 1 as a consequence of Theorem 2. The space of normal functions $N$ is the set of all $f \in H(D)$ with the property that $\left\{f \circ \varphi_{b}\right.$ : $b \in D\}$ is a normal family of holomorphic functions.

LEMMA 2. Let $f$ be a normal function on $D$ and $a \in \partial D$. If $R(f, a) \neq \mathbf{C}$ then for each $r>0$ there exist a sequence $\left\{a_{n}\right\}$ in $\Delta(a, r)$, an $a^{\prime} \in \partial D$ with $\left|a^{\prime}-a\right|<r$, and $a w_{0} \in \mathbf{C}$ satisfying $f \circ \varphi_{a_{n}} \rightarrow w_{0}$ in $H(D)$.

Proof. Assume first that $f\left(\Delta\left(a, r_{1}\right)\right)=\mathbf{C} \backslash\left\{w_{0}\right\}$ for some $0<r_{1}<r$ and $w_{0} \in \mathbf{C}$. Then there exists $\left\{a_{n}\right\}$ in $\Delta\left(a, r_{1}\right)$ converging to $a^{\prime} \in \partial D$ and satisfying $f\left(a_{n}\right) \rightarrow w_{0}$. Since $\left\{f \circ \varphi_{a_{n}}\right\}$ is a normal family we may assume without loss of generality that there is a function $f_{0} \in H(D)$ with $f_{n}=f \circ \varphi_{a_{n}}$ converging to $f_{0}$ in $H(D)$. Since

$$
f_{0}(0)=\lim _{n \rightarrow \infty} f_{n}(0)=\lim _{n \rightarrow \infty} f\left(a_{n}\right)=w_{0}
$$

and since there is a neighborhood of 0 in which each $f_{n}$ omits the value $w_{0}$ it follows from Hurwitz's Theorem that $f_{0} \equiv w_{0}$.

Finally, if $f$ satisfies the hypothesis and omits two or more points in $\mathrm{C}$ then by a known result $f$ has finite nontangential limits on a dense subset of $\partial D \cap \partial \Delta(a, r)$, see Corollary 22.1 [5] for example. The sequence $\left\{a_{n}\right\}$ can be chosen to converge nontangentially to any of these points, and a standard argument gives the result. This completes the proof of the lemma. 
REMARK. By taking $M(a, r)=M \cap N$ we see that the lemma implies that hypothesis (i) of Theorem 2 follows from the corresponding hypothesis of Theorem 1.

Recall that for $a \in D$ the function $b_{a}(z)$ is the Blaschke product $z \varphi_{a}(z)$.

LEMMA 3. Given $0<s<1$ there is a $r=r(s)$ with $0<r<1$ and such that for any $a \in D$ :

$$
\begin{aligned}
& \text { (i) } D(0, s) \Subset b_{a}(D(0, r)) \text { and } \\
& \text { (ii) }\left|b_{a}(z)\right|>s \text { for all }|z|=r \text {. }
\end{aligned}
$$

Proof. Let $0<s<1$ and $a \in D$. Pick $t=t(a, s)$ to be the smallest positive number satisfying $\left|b_{a}(z)\right| \geq s$ for all $|z|=t$. It is evident that the function $t=t(a)$ is continuous for $a \in D$. Furthermore, $\bar{a} b_{a}(z)$ tends to $z$ as $|a|$ tends to 1 for any $z \in D$. As a result, $t(a, s)$ tends to $s$ as $|a|$ tends to 1 and hence

$$
s \leq t_{s}=\sup \{t(a, s): a \in D\}<1 .
$$

Finally, let $r$ lie in the interval $\left(t_{s}, 1\right)$. Since $\left|b_{a}(z)\right|=1$ for $z \in \partial D$ and $\left|b_{a}(z)\right| \geq s$ for $|z|=t(a, s)$, we have that $\left|b_{a}(z)\right|>s$ for $t(a, s)<|z|$ $\leq 1$. In other words, statement (ii) is true. But $b_{a}(0)=0$ and hence the argument principle implies that the closure of $D(0, s)$ is contained in $b_{a}(D(0, r))$ and the proof is complete.

Using Lemma 3 we can prove that the hypotheses (ii)(a) and (ii)(b) of Theorem 1 imply the corresponding hypothesis (ii) of Theorem 2 and hence complete the proof of Theorem 1.

By the Banach-Steinhaus Theorem for $F$-spaces, see Theorem 2.6 [9], it follows from (ii)(a) that there is a constant $K<\infty$ with

$$
\left\|f \circ b_{a}\right\| \leq K\|f\|, \quad f \in M, a \in D .
$$

By (ii)(b) there is a function $f$ in $M$ with $f(D)=$ C. Fix $\varepsilon>0$ and $R>0$. Since scalar multiplication is a continuous operation in an $F$-space we may assume that $\|f\|<\varepsilon / K$. Choose $0<s<1$ with $D(0, R) \Subset$ $f(D(0, s))$ and using Lemma 3 pick $r$ satisfying (3). Taking $\eta=$ $\inf \{\delta(w, f, D(0, s)):|w| \leq R\}$ we claim that hypothesis (ii) of Theorem 2 is satisfied with $r(R, \varepsilon)=r$ and $\eta(R, \varepsilon)=\eta$.

Given $a \in D$ we take $f_{a}=f \circ b_{a}$. Note that $f_{a} \circ \varphi_{a}=f_{a}$ since $\varphi_{a}$ is its own inverse. Using the remarks following Lemma 1 we conclude that

$$
\begin{aligned}
\delta\left(w, f_{a} \circ \varphi_{a}, D(0, r)\right) & =\delta\left(w, f \circ b_{a}, D(0, r)\right) \\
& =\delta\left(w, f, b_{a}(D(0, r))\right) \geq \eta, \quad|w| \leq R .
\end{aligned}
$$


Since $\left\|f_{a}\right\|<\varepsilon$ by (4), we have shown that hypothesis (ii) holds and the proof is complete.

REMARK 3. The above argument also reveals a variant on hypothesis Theorem 1 (ii)(b), namely,

(ii)(b)': for every $R>0, \varepsilon>0$, there exists $f$ in $M$ with $\|f\|<\varepsilon$ and $D(0, R) \Subset f(D)$.

We combine the proof of Theorem 1 with an idea in the Brown and Hansen paper mentioned in the introduction to obtain:

THEOREM 3. Let $M$ be an F-space in $H(D)$ that satisfies hypothesis of Theorem 2(ii). Then the set of all $f$ in $M$ for which $R(f, a)$ omits at most one point for every $a \in \partial D$ is a dense $G_{\delta}$-set.

Proof. Let $\left\{a_{j}\right\}$ be countable dense subset of $\partial D$. For positive integers, $j, k$, and $m$ let $A(j, k, m)=\left\{f \in M\right.$ : there exists $\left|w_{1}\right| \leq m$, $\left|w_{2}\right| \leq m$, with $\left|w_{1}-w_{2}\right| \geq m^{-1}$ and $w_{i} \notin f\left(\Delta\left(a_{j}, k^{-1}\right)\right)$ for $\left.i=1,2\right\}$. By the Baire category theorem, it suffices to prove that each $A(j, k, m)$ is a closed and nowhere dense set.

That $A(j, k, m)$ is a closed set follows as in [4]. Let $g \in A(j, k, m)$ and $\varepsilon>0$. Since $g\left(\Delta\left(a_{j}, k^{-1}\right)\right)$ omits two points it must satisfy hypothesis Theorem 2 (i)(b) with $a=a_{j}$ as was shown in Lemma 2. Since hypothesis (ii) of Theorem 2 is assumed to be true it follows from the proof of Theorem 2 that there exists an $f$ in $M$ with $\|f\|<\varepsilon$ and $D(0, m) \Subset$ $(g+f)\left(\Delta\left(a_{j}, k^{-1}\right)\right)$. In particular, $g+f$ is not in $A(j, k, m)$. Since $\varepsilon$ is arbitrary this means that $A(j, k, m)$ is nowhere dense and the proof is complete.

REMARK 4. It seems reasonable that the one-point exception could be removed in the above theorem.

\section{Several Examples.}

EXAMPLE 1. The spaces BMOA and $B$ both satisfy the hypothesis of Theorem 1 . The metrics for these $F$-spaces are respectively (cf. [2], [1]):

$$
\begin{aligned}
\|f\|_{\text {ВMOA }} & =|f(0)|+\sup _{a \in D}\left\{\frac{1}{2 \pi} \int_{0}^{2 \pi}\left|f \circ \varphi_{a}\left(e^{\imath \theta}\right)-f(a)\right|^{2} d \theta\right\}^{1 / 2} \\
\|f\|_{B} & =|f(0)|+\sup _{a \in D}\left|\left(f \circ \varphi_{a}\right)^{\prime}(0)\right| .
\end{aligned}
$$


In addition, the space of normal functions $N$ can also be characterized by the finiteness of the quantity

$$
\sup _{a \in D} \frac{\left|\left(f \circ \varphi_{a}\right)^{\prime}(0)\right|}{1+|f(a)|^{2}}<\infty
$$

and hence BMOA $\subset B \subset N$. So BMOA and $B$ satisfy the hypothesis of Theorem 1 (i). The composition operators $C_{a}$ are contractions on BMOA, see $\$ 4$ [12], and as a consequence of the Schwarz-Pick lemma also on $B$. Thus hypothesis (ii)(a) of Theorem 1 is satisfied for these two spaces. Finally, we need a function in BMOA which maps onto $\mathbf{C}$ in order to complete the verification that BMOA and $B$ satisfy the hypothesis of Theorem 1. However this is a well known result; see [13, p. 252] for a geometric construction.

We note that while the space of normal functions has similar properties to BMOA and $B$ it is not a linear space. By a result of Lappin [8] the sum of two normal functions need not be a normal function.

Example 2. The Hardy spaces $H^{p}, 0<p<\infty$, are determined by the finiteness of (cf. [7])

$$
\|f\|_{p}=\sup _{r<1}\left\{\frac{1}{2 \pi} \int_{0}^{2 \pi}\left|f\left(r e^{i \theta}\right)\right|^{p} d \theta\right\}^{1 / p} .
$$

For $p \geq 1$, these spaces are Banach spaces and for $0<p<1$, they are $F$-spaces with metric induced by $\|\cdot\|_{p}^{p}$. It is well known that BMOA is a dense subspace of each of these spaces and hence the conclusion of Theorem 2 holds by Remark 2 (i).

Closely related to these spaces is the Nevanlinna space of functions of bounded characteristic. Here the metric is defined by (cf. [10])

$$
d(f)=\sup _{r<1}\left\{\frac{1}{2 \pi} \int_{0}^{2 \pi} \log \left(1+\left|f\left(r e^{i \theta}\right)\right|\right) d \theta\right\} .
$$

The resulting topology is stronger than that of $H(D)$ and addition of functions is a continuous operation. However, as observed in [6, p. 146], scalar multiplication is not a continuous operation so the Nevanlinna space is not an $F$-space.

Nevertheless, the hypotheses of Theorem 2 are valid since nontangential limits exist almost everywhere and BMOA is a subspace. Thus, by Remark 2 (ii) the conclusion of Theorem 2 is valid for this space. 
EXample 3. The Dirichlet spaces, $D_{\alpha}$ for $0 \leq \alpha<1$, are defined by the norm

$$
\|f\|_{\alpha}=|f(0)|+\left\{\iint_{D}\left|f^{\prime}(z)\right|^{2}\left(1-|z|^{2}\right)^{1-2 \alpha} d m_{2}(z)\right\}^{1 / 2}
$$

where $m_{2}$ denotes area measure, see [11]. If $f(z)=\sum a_{n} z^{n}$, then $f$ is in $D_{\alpha}$ if and only if the comparable norm $\left\{\sum\left(1+n^{2}\right)^{\alpha}\left|a_{n}\right|^{2}\right\}^{1 / 2}$ is finite.

Clearly, $D_{\alpha} \cap H^{\infty}$ (and hence $D_{\alpha} \cap N$ ) is dense in $D_{\alpha}$. Let $a \in D$, then using the Schwarz lemma and the fact that $b_{a}(z)$ is a 2-to-1 mapping of $D$ onto itself with $b_{a}(0)=0$ yields

$$
\begin{aligned}
& \left\|C_{a} f\right\|_{\alpha}=|f(0)|+\left\{\iint_{D}\left|f^{\prime}\left(b_{a}(z)\right)\right|^{2}\left(1-|z|^{2}\right)^{1-2 \alpha}\left|b_{a}^{\prime}(z)\right|^{2} d m_{2}(z)\right\}^{1 / 2} \\
& \quad \leq|f(0)|+\left\{\iint_{D}\left|f^{\prime}\left(b_{a}(z)\right)\right|^{2}\left(1-\left|b_{a}(z)\right|^{2}\right)^{1-2 \alpha}\left|b_{a}^{\prime}(z)\right|^{2} d m_{2}(z)\right\}^{1 / 2} \\
& \quad \leq|f(0)|+\left\{\iint_{D}\left|f^{\prime}(w)\right|^{2}\left(1-|w|^{2}\right)^{1-2 \alpha} 2 d m_{2}(w)\right\}^{1 / 2} \\
& \quad \leq \sqrt{2}\|f\|_{\alpha} .
\end{aligned}
$$

Thus, with the possible exception of (ii)(b), the hypotheses to Theorem 1 are satisfied.

For $\alpha \geq 1 / 2$, each function in $D_{\alpha}$ must have range with finite area because the Dirichlet integral (essentially $\|f\|_{1 / 2}^{2}$ ) must be finite. Therefore the conclusion to the theorem cannot possibly hold; see [14] for an analysis of the boundary behavior for the Dirichlet space $D_{1 / 2}$. For the range $0 \leq \alpha<1 / 2$ we will show that $D_{\alpha}$ contains onto functions and hence the conclusion to Theorem 1 holds for these spaces also.

LeMma 4. For $0 \leq \alpha<1 / 2, D_{\alpha}$ contains a function mapping onto $\mathbf{C}$.

Proof. Fix $0 \leq \alpha<1 / 2$ and let $0<\beta<\frac{1}{2}(1-\alpha)$. Using the binomial theorem we see that $g(z)=(1-z)^{-\beta}-1$ is in $D_{\alpha}$. The function $g$ is a schlicht function mapping $D$ onto a region containing the sector $S=\left\{w:|\operatorname{Arg} w|<\frac{1}{2} \pi \beta\right\}$. By choosing the least integer $n>2 / \alpha$, the function $f(z)=z g\left(z^{n}\right)$ is also in $D_{\alpha}$. We leave as an exercise the fact that the sector $S$ is contained in the range of $f$. Having said that, we are done, since $f(\lambda z)=\lambda f(z)$ for any $n$th root of unity implies $f$ maps onto $\mathbf{C}$. 
EXAMPLE 4. The space $H(D)$ is an $F$-space and satisfies the hypothesis of Theorem 1.

ExAmple 5. The "little" Bloch space, denoted $B_{0}$, is the space of analytic functions for which

$$
\lim _{|z| \rightarrow 1}\left|f^{\prime}(z)\right|^{2}\left(1-|z|^{2}\right)=0 .
$$

With the exception of (ii)(b) the hypotheses of Theorem 1 can be verified as with $B$. Fix $R>0, \varepsilon>0$ and let $g \in B$ satisfy $\|g\|_{B}<\varepsilon$ and $g(D)=C$. Clearly, $g_{r}(z)=g(r z)$ satisfies $D(0, R) \Subset g_{r}(D)$ for some $r<1$ and hence by Remark 3 the conclusion to Theorem 1 holds for $B_{0}$. Observe that the existence of a $B_{0}$-function mapping onto $C$ is now a consequence of the Baire category theorem.

As a last example we give a simple class of spaces for which Theorem 3 applies but not Theorem 2 .

EXAMPLE 6. Let $h(r)$ be a nondecreasing function on $[0,1)$ with $h(0)=1$. Let $M_{h}$ be the space of holomorphic functions for which

$$
\|f\|_{h}=\sup _{|z|<1} \frac{|f(z)|}{h(|z|)}
$$

is finite. These spaces satisfy the hypothesis of Theorem 3 but do not satisfy any "nice" boundary conditions as in Theorem 2 (i).

\section{REFERENCES}

[1] J. M. Anderson, J. Clunie, and Ch. Pommerenke, On Bloch functions and normal functions, J. Reine Angew. Math., 270 (1974), 12-37.

[2] A. Baernstein II, Analytic Functions of Bounded Mean Oscillation, "Aspects of Contemporary Complex Analysis, Proceedings of the NATO Advanced Study Institute at Durham, 1979, (ed. D. A. Brannan and J. G. Clunie), Academic Press, London, 1980.

[3] D. D. Bonar and R. W. Carroll, On the range sets of $H^{p}$ functions, Math. Z., 154 (1977), 261-263.

[4] L. Brown and L. Hansen, On the range sets of $H^{p}$ functions, Pacific J. Math., 42 (1972), 27-32.

[5] E. F. Collingwood and M. L. Cartwright, Boundary theorems for a function meromorphic in the unit circle, Acta Math., 87 (1952), 83-146.

[6] C. S. Davis, Iterated limits in $N^{*}\left(U^{n}\right)$, Trans. Amer. Math. Soc., 178 (1973), 139-146.

[7] K. Hoffman, Banach Spaces of Analytic Functions, Prentice Hall, Englewood Cliffs, New Jersey, 1962. 
[8] P. A. Lappin, Jr., Non-normal sums and products of unbounded normal functions, Michigan Math. J., 8 (1961), 187-192.

[9] W. Rudin, Functional Analysis, McGraw-Hill, New York, 1973.

[10] J. H. Shapiro and A. L. Shields, Unusual topological properties of the Nevanlinna class, Amer. J. Math., 97 (1976), 915-936.

[11] D. A. Stegenga, Multipliers of the Dirichlet space, Illinois J. Math., 24 (1980), 113-139.

[12] K. Stephenson, Weak subordination and stable classes of meromorphic functions, Trans. Amer. Math. Soc., 262 (1980), 565-577.

[13] D. A. Stegenga and K. Stephenson, A geometric characterization of analytic functions with bounded mean oscillation, J. London Math. Soc., (2) 24 (1981), 243-254.

[14] _ Generic covering properties for spaces of analytic functions, Pacific J. Math., 119 (1985), 227-243.

Received July 31,1985 . Both authors are supported by grants from the National Science Foundation.

UNIVERSITY OF HAWAII

HONOLULU, HI 96822

AND

UNIVERSITY OF TENNESSEE

KNOXVILLE, TN $37996-1300$ 



\section{PACIFIC JOURNAL OF MATHEMATICS EDITORS}

\author{
V. S. VARADARAJAN \\ (Managing Editor) \\ University of California \\ Los Angeles, CA 90024 \\ Herbert Clemens \\ University of Utah \\ Salt Lake City, UT 84112 \\ R. FINN \\ Stanford University \\ Stanford, CA 94305
}

\section{HERMANN FLASCHKA}

University of Arizona

Tucson, AZ 85721

RAMESH A. GANGOLLI

University of Washington

Seattle, WA 98195

VAUGHAN F. R. JONES

University of California

Berkeley, CA 94720

ROBION KIRBY

University of California

Berkeley, CA 94720
C. C. MOORE

University of California

Berkeley, CA 94720

H. SAMELSON

Stanford University

Stanford, CA 94305

HAROLD STARK

University of California, San Diego

La Jolla, CA 92093

\section{ASSOCIATE EDITORS}
R. ARENS
E. F. BECKENBACH
B. H. NEUMANN
F. WOLF
K. YOSHIDA
(1906-1982)

\section{SUPPORTING INSTITUTIONS}

UNIVERSITY OF ARIZONA UNIVERSITY OF OREGON

UNIVERSITY OF BRITISH COLUMBIA UNIVERSITY OF SOUTHERN CALIFORNIA

CALIFORNIA INSTITUTE OF TECHNOLOGY STANFORD UNIVERSITY

UNIVERSITY OF CALIFORNIA

MONTANA STATE UNIVERSITY

UNIVERSITY OF HAWAII

UNIVERSITY OF NEVADA, RENO

UNIVERSITY OF TOKYO

NEW MEXICO STATE UNIVERSITY

UNIVERSITY OF UTAH

OREGON STATE UNIVERSITY

WASHINGTON STATE UNIVERSITY

UNIVERSITY OF WASHINGTON 


\section{Pacific Journal of Mathematics}

\section{Vol. 126, No. $1 \quad$ November, 1987}

John Dauns, Uniform dimensions and subdirect products $\ldots \ldots \ldots \ldots \ldots 1$

William B. Jacob, Quadratic forms over dyadic valued fields. I. The graded

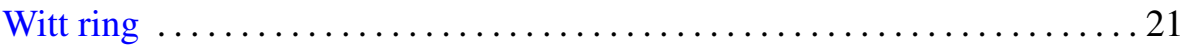

Michael R. Kelly, Minimizing the number of fixed points for self-maps of

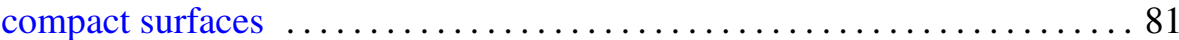

Edward Kissin, On some reflexive operator algebras constructed from two sets of closed operators and from a set of reflexive operator algebras . . 125

Robert Langlands, The Dirac monopole and induced representations . . . . . 145

David A. Stegenga and Kenneth R. Stephenson, Generic covering properties for spaces of analytic functions. II $\ldots \ldots \ldots \ldots \ldots \ldots \ldots 153$

Gerard Alan Venema, Approximating codimension two embeddings of cells 165

Peter Waksman, Determining an analytic function from its distribution of values 